\title{
Scaling of space-time modes with Reynolds number in two-dimensional turbulence
}

\author{
By N. K. -R. KEVLAHAN ${ }^{1}$, J. ALAM ${ }^{1}$ AND O. V. VASILYEV \\ ${ }^{1}$ Department of Mathematics \& Statistics, McMaster University, Hamilton L8S 4K1, Canada \\ ${ }^{2}$ Department of Mechanical Engineering, University of Colorado, Boulder, CO 80309, USA
}

(Received 2 May 2006 and in revised form 28 September 2006)

It has been estimated that the number of spatial modes (or nodal values) required to uniquely determine a two-dimensional turbulent flow at a specific time is finite, and is bounded by $R e^{4 / 3}$ for forced turbulence and $R e$ for decaying turbulence. The usual computational estimate of the number of space-time modes required to calculated decaying two-dimensional turbulence is $\mathscr{N} \sim R e^{3 / 2}$. These bounds neglect intermittency, and it is not known how sharp they are. In this paper we use an adaptive multi-scale wavelet collocation method to estimate for the first time the number of space-time computational modes $\mathscr{N}$ necessary to represent two-dimensional decaying turbulence as a function of Reynolds number. We find that $\mathscr{N} \sim R e^{0.9}$ for $1260 \leqslant R e \leqslant 40400$ over many eddy turn-over times, and that temporal intermittency is stronger than spatial intermittency. The spatial modes alone scale like $R e^{0.7}$. The $\beta$-model then implies that the spatial fractal dimension of the active regions is 1.2 , and the temporal fractal dimension is 0.3 . These results suggest that the usual estimates are not sharp for adaptive numerical simulations. The relatively high compression confirms the importance of intermittency and encourages the search for reduced mathematical models of two-dimensional turbulence (e.g. in terms of coherent vortices).

\section{Introduction}

Turbulence is difficult to approximate mathematically, and to calculate numerically, because it is active over a large and continuous range of length scales (e.g. from less than a millimetre to hundreds of kilometres in the atmosphere). The range of active scales increases with Reynolds number (like $R e^{3 / 4}$ for three-dimensional turbulence and $R e^{1 / 2}$ for two-dimensional turbulence) which means flows are increasingly difficult to calculate at the large Reynolds numbers of practical interest. Although the active flow regions extend over many scales, they are distributed inhomogeneously in both space and time. This inhomogeneity is called intermittency. Exploiting intermittency to construct reduced models of turbulence (e.g. in terms of coherent vortices), or to optimally use a finite number of computational elements (e.g. using adaptive mesh refinement) are promising ways of constructing a simplified representation of turbulence (e.g. Farge, Schneider \& Kevlahan 1999; Kevlahan \& Vasilyev 2005).

The solutions of partial differential equations generally have an infinite number of degrees of freedom. However, Foias \& Prodi (1967) conjectured that solutions of the Navier-Stokes equations are determined uniquely by a finite number of spatial modes at each time. This conjecture was refined by Foias \& Temam (1984), Constantin (1985) and Constantin, Foias \& Temam (1988) before being proved in the case of forced two-dimensional turbulence with periodic boundary conditions by Friz \& Robinson 
(2001). These results were recently extended to three-dimensional flow past a body by Galdi (2006), who showed that such flows are uniquely determined by the velocity at a finite number of nodal points near the body.

The finiteness of turbulence can be understood in several ways: in terms of the dimension of its attractor, the number of modes (e.g. eigenfunctions), or the number of nodes at which it is necessary to know the velocity in order to uniquely determine the flow. Constantin et al. (1988) found an upper bound for the number of Fourier modes $\mathscr{N}$ required to represent the forced two-dimensional Navier-Stokes equations,

$$
\mathscr{N} \sim G r^{2 / 3}(1+\log (G r))^{1 / 3} \approx G r^{2 / 3},
$$

where $G r$ is the generalized Grashof number, which is $O\left(R e^{2}\right)$ as $G r \rightarrow \infty$ (Doering \& Foias 2002). This estimate is therefore equivalent to $\mathscr{N} \sim R e^{4 / 3}$, which is significantly higher than the usual computational estimate $\mathscr{N} \sim \operatorname{Re}$ (based on a homogeneous minimum length scale $l_{\eta} \sim R e^{-1 / 2}$ ). Note that the equivalent space-time computational estimate is $\mathscr{N} \sim R e^{3 / 2}$ (which simply assumes a time step $\Delta t \approx 1 / l_{\eta}$ ). The central goal of the present paper is to understand better the scaling of $\mathscr{N}$ as a function of Reynolds number, i.e. to estimate $\alpha$ in the relation

$$
\mathscr{N} \sim R e^{\alpha}
$$

where $\mathscr{N}$ is the number of either spatial degrees of freedom, or space-time degrees of freedom of the turbulence. By degrees of freedom, we mean computational degrees of freedom which differs from the true mathematical number of degrees of freedom due to the finite error tolerance used here.

Paladin \& Vulpiani (1987) used the $\beta$-model (Frisch, Nelkin \& Sulem 1978) to show that the spatial degrees of freedom of an intermittent turbulent flow should scale like

$$
\mathscr{N} \sim \operatorname{Re}^{3 D_{F} /\left(D_{F}+1\right)},
$$

where $D_{F} \leqslant 3$ is the fractal dimension of the active part of the flow. A similar calculation for two-dimensional turbulence gives

$$
\mathscr{N} \sim \operatorname{Re}^{3 D_{F} /\left(D_{F}+4\right)} .
$$

Measurements of the exponent $\alpha$ in equation (1.2) can therefore be used together with (1.3) or (1.4) to estimate the fractal dimension, and hence the intermittency, of the active regions of the flow. Spatial intermittency of three-dimensional turbulence is associated with $D_{F}<3$ and $\alpha<9 / 4$ (and $D_{F}<2$ and $\alpha<1$ for two-dimensional turbulence).

Yakhot \& Sreenivasan (2005) recently suggested that intermittency may actually increase the number of computational degrees of freedom if the strongest (and rarest) fluctuations are resolved. They estimate that the total number of space-time degrees of freedom in three-dimensional turbulence is actually $\mathscr{N} \sim R e^{4}$, rather than the usual estimate of $\mathscr{N} \sim R e^{3}$ (e.g. Frisch 1995). They note that $\mathscr{N}$ could in fact be smaller than $R e^{3}$ if only the 'interesting' parts of the flow were resolved, since their estimate assumes a uniform computational grid. However, they are pessimistic that this can be done: "Even if the interesting parts of a turbulent flow are not space-filling [...] we do not yet know how to track them efficiently in hydrodynamic turbulence." This is precisely what we attempt to do in the present paper.

Mathematical analysis has confirmed our intuition that turbulence is finitely representable. However, these estimates do not take into account spatial intermittency, and are therefore unlikely to be optimal. In addition, the analysis neglects the temporal degrees of freedom. In this paper we attempt to address both issues: we estimate 
the number of space-time modes necessary to compute two-dimensional decaying turbulence using a method that takes into account intermittency. Our approach uses the space-time adaptive wavelet numerical method described in Alam, Kevlahan \& Vasilyev (2006). This method automatically refines the computational grid in both space and time in order to resolve the two-dimensional Navier-Stokes equations to the desired accuracy with the minimum number of computational elements (i.e. it produces an optimal $\mathscr{N}$-term approximation). The method uses local time steps (i.e. the time step varies with position) as well as an adaptive spatial grid. In addition, the global time integration error is controlled, something not possible using conventional time marching methods. By counting the number of significant wavelets we obtain an estimate of the number of nodal values or modes required to represent the flow to the desired accuracy. To our knowledge this is the first attempt to find an adaptive computational estimate of the scaling of the space-time (or spatial) modes of a turbulent flow.

Although turbulence is essentially three-dimensional in nature, two-dimensional turbulence retains many important properties (such as coherent structures and intermittency) and its relative computational simplicity makes it a suitable test case for new theories and computational techniques. In addition, the best mathematical upper bounds exist only for the two-dimensional Navier-Stokes equations (e.g. Friz \& Robinson 2001; Jones \& Titi 1993). Note that, due to the lack of vortex stretching, two-dimensional turbulence is usually considered to be less intermittent than threedimensional turbulence. However, we will see that this is not necessarily the case. We consider decaying two-dimensional turbulence since forcing is non-trivial in a space-time approach, and different forcing schemes could lead to different results (Tran, Shepherd \& Cho 2004). Because energy remains bounded as $R e \rightarrow \infty$ in the decaying case, we should not necessarily expect to observe the scaling given by (1.1). Differences may arise because the forced case has both an enstrophy cascade to small scales and an inverse energy cascade to large scales, while the decaying case has only the former. However, we might expect that intermittency is associated primarily with the small scales of the enstrophy cascade.

\section{Space-time adaptive wavelet simulation}

As pointed out by Yakhot \& Sreenivasan (2005), in order to count the number of active modes in a turbulent flow we need a way of detecting and tracking them. Computationally, this is an adaptive mesh problem: we must put the computational nodes (or modes) where they are needed (in space and time) in order to compute the flow to a prescribed tolerance. This means we need dynamic adaptive grids and local time steps. The number of grid points is then an approximation to the number of nodes, modes or degrees of freedom necessary to represent the flow. By repeating the calculation for a sequence of Reynolds numbers we can estimate the exponent $\alpha$ in equation (1.2). We use the space-time adaptive wavelet collocation (STAWCM) method introduced in Alam et al. (2006) to achieve the necessary adaptivity. In this section we briefly outline the main features of this method, especially regarding the problem of estimating degrees of freedom. Note that in the AWCM a unique wavelet corresponds to each grid point (or node), and so the number of modes is equal to the number of nodes. The number of nodes should be of the same order as the number of degrees of freedom required to represent the flow. In the following, position refers to a point in space-time, i.e. $\boldsymbol{x}=(x, y, t) \in \mathbb{R}^{3}$. It is important to remember that the spatial and temporal grid are adapted simultaneously and in the same way. Alam et al. (2006) provides details of the STAWCM method. 
STAWCM is based on a multi-resolution approximation (MRA). In this MRA functions are approximated using tensor product second-generation wavelets (Vasilyev $2003)$ on a nested set $\left(G^{j} \subset G^{j+1}\right)$ of grid points

$$
G^{j}=\left\{\boldsymbol{x}_{\boldsymbol{k}}^{j} \in \Omega: \boldsymbol{x}_{\boldsymbol{k}}^{j}=\boldsymbol{x}_{2 \boldsymbol{k}}^{j+1}, \boldsymbol{k} \in \mathscr{K}^{j}, j \in \mathscr{Z}\right\},
$$

where $\boldsymbol{k}=\left(k_{1}, \ldots, k_{n}\right)$ is the position in $n$-dimensional space, $j$ is the level (or scale), $\boldsymbol{x}_{\boldsymbol{k}}^{j}$ are the grid points in the computational domain $\Omega$, and $\mathscr{Z}$ and $\mathscr{K}^{j}$ are some suitable index sets.

To illustrate the discretization of partial differential equations, let us consider the MRA of a function $u(\boldsymbol{x})$,

$$
u(\boldsymbol{x})=\sum_{\boldsymbol{l} \in \mathscr{L}^{0}} c_{\boldsymbol{l}}^{0} \phi_{l}^{0}(\boldsymbol{x})+\sum_{j=0}^{+\infty} \sum_{\mu=1}^{2^{n}-1} \sum_{\boldsymbol{k} \in \mathscr{K}^{\mu, j}} d_{\boldsymbol{k}}^{\mu, j} \psi_{\boldsymbol{k}}^{\mu, j}(\boldsymbol{x}),
$$

where $\phi_{l}^{0}(\boldsymbol{x})$ and $\psi_{k}^{\mu, j}$ are, respectively, the $n$-dimensional scaling functions and wavelets of different families $\mu$ and levels of resolution $j$. The strength of the wavelet decomposition (2.2) is that the coefficients $d_{\boldsymbol{k}}^{\mu, j}$ measure the variation of $u(x)$ at scale $2^{-j}$ near position $\boldsymbol{x}_{\boldsymbol{k}}^{j}$. These coefficients decay rapidly in smooth regions, and are large only in regions where the gradient of $u(x)$ is large. This suggests that there exists a decreasing sequence of positive numbers $\epsilon_{J}$ such that $\forall j \geqslant J, \quad\left|d_{k}^{\mu, j}\right|<\epsilon_{J}$. In other words, any truncation of the above sum over $j$ is an approximation of $u(x)$ at scale $2^{-j}$. Also, if $u(x)$ is smooth, except at some isolated points, the above truncation requires a small number of coefficients to approximate $u(x)$. Thus, the MRA not only approximates a function, it also compresses it.

For intermittent functions, $d_{k}^{\mu, j}$ is large only at those positions $\boldsymbol{k}$ where the function has a strong gradient. Therefore, discarding coefficients whose magnitudes are smaller than a given threshold $\epsilon$ truncates the infinite sum (2.2) to a finite sum, as well as compressing the function. The truncated sum $u^{J}(\boldsymbol{x})$ is a good approximation of $u(x)$ at level $J$ in the weighted residual sense, i.e.

$$
\int_{\Omega}\left(u(\boldsymbol{x})-u^{J}(\boldsymbol{x})\right) \delta\left(\boldsymbol{x}-\boldsymbol{x}_{k}^{J}\right) \mathrm{d} \boldsymbol{x}=0 .
$$

This restriction establishes a one-to-one correspondence between $d_{k}^{\mu, j}$ and $\boldsymbol{x}_{k}^{J}$. The grid adaptation strategy is based on the fact that discarding a wavelet coefficient is equivalent to discarding the corresponding collocation point. To construct a grid adapted to an intermittent solution we collect all collocation points $\boldsymbol{x}_{k}^{j}$ such that $\left|d_{k}^{\mu, j}\right| \geqslant \epsilon$; i.e.

$$
G_{\epsilon}^{j}=\left\{\boldsymbol{x}_{\boldsymbol{k}}^{j} \in \Omega: \boldsymbol{x}_{\boldsymbol{k}}^{j}=\boldsymbol{x}_{2 \boldsymbol{k}}^{j+1}, k \in \mathscr{K}^{j}, j \in \mathscr{Z},\left\|d_{\boldsymbol{k}}^{\mu, j}\right\|_{p} \geqslant \epsilon\|u\|_{p}\right\} .
$$

The adaptive wavelet decomposition then takes the form

$$
u_{\epsilon}^{J}(\boldsymbol{x})=\sum_{\boldsymbol{l} \in \mathscr{L}^{0}} c_{l}^{0} \phi_{l}^{0}(\boldsymbol{x})+\sum_{j=0}^{+\infty} \sum_{\mu=1}^{2^{n}-1} \sum_{\substack{\boldsymbol{k} \in \mathfrak{x}^{\mu} \mu, j \\\left\langle d_{k}^{\mu, j}, \|_{p} \in \in|u| p\right.}} d_{\boldsymbol{k}}^{\mu, j} \psi_{\boldsymbol{k}}^{\mu, j}(\boldsymbol{x}),
$$

For functions with localized structure in $\Omega, G_{\epsilon}^{j}$ is much smaller than $G^{j}$ for all $j$. Derivatives can then be calculated on $G_{\epsilon}$ using the appropriate finite difference formula (Vasilyev 2003). All operations have computational complexity $O(\mathscr{N})$, where $\mathscr{N}$ is the number of significant wavelet modes. 
The approximation error is controlled by the tolerance parameter $\epsilon$,

$$
\left\|u(\boldsymbol{x})-u_{\epsilon}^{J}(\boldsymbol{x})\right\|_{p} \sim \epsilon\|u\|_{p}, \quad \epsilon \rightarrow 0 .
$$

Relation (2.6) is independent of the dimensionality of the problem. Since the number of active grid points depends on the dimension and the order of wavelets being used, one can show that the number of active coefficients satisfies

$$
\mathscr{N} \sim \epsilon^{-n / p},
$$

where $p$ is the order of wavelets used and $n$ is the dimensionality of the problem ( $p=6$ and $n=3$ in this paper). In other words, the truncation error is related to the number of terms retained as

$$
\left\|u(\boldsymbol{x})-u_{\epsilon}^{J}(\boldsymbol{x})\right\|_{p} \sim \mathscr{N}^{-p / n} .
$$

The AWCM described above is the basis of an adaptive multilevel solver for the Navier-Stokes equations, which are formulated as a pseudo-boundary value problem in a space-time domain $\Omega=D \times(0, T)$ where $D \subset \mathbb{R}^{2}$ and $T \in \mathbb{R}^{+}$. For technical reasons (limited memory) $T$ is split into several subdomains. The AWCM finds the solution to the Navier-Stokes equations with the smallest number of space-time grid points possible given a specified residual error $\epsilon$. This is called an optimal $\mathscr{N}$-term approximation. We claim that $\mathscr{N}$ is therefore a good practical upper bound on the number of space-time degrees of freedom of the flow. Alam et al. (2006) and Vasilyev \& Kevlahan (2005) provide details of the AWCM space-time and time marching (adaptive in space only) methods.

\section{Numerical simulations}

The initial conditions are specified in Fourier space, and approximate a fully developed two-dimensional turbulent flow with specified power-law energy spectrum and random phases,

$$
\hat{\boldsymbol{u}}(\boldsymbol{k}, 0)=\frac{\left(-k_{y}, k_{x}\right)}{|\boldsymbol{k}|} \sqrt{\frac{E(k)}{2 \pi|\boldsymbol{k}|}} \exp (\mathrm{i} 2 \pi \varphi),
$$

where $\varphi$ are random phases and $E(k)=g_{2} k^{4} /\left(g_{1}+k^{2}\right)^{7 / 2} \exp \left(-k^{2} / k_{\max }^{2}\right)$ is the energy spectrum. Note that $E(k) \sim k^{4}$ for small wavenumbers, $E(k) \sim k^{-3}$ for intermediate wavenumbers, and the spectrum decays exponentially at large wavenumbers; $\hat{\boldsymbol{u}}$ is divergence free by construction. This spectrum is similar to the $k^{-3.3}$ spectrum measured experimentally for two-dimensional soap film turbulence by Martin et al. (1998). The constants $g_{1}$ and $g_{2}$ are chosen such that the initial r.m.s. velocity is $U=4.65$ and the initial integral length scale is $L=1.09$ for all simulations. The velocity field is transformed to physical space to specify to initial time boundary condition of the space-time domain. Simulation parameters are summarized in table 1.

All simulations are performed in the space-time computational domain $[0,2 \pi] \times$ $[0,2 \pi] \times[0,128]$, where the spatial domain is a doubly periodic square and the time interval is $[0,128]$ (time is normalized by the initial eddy turn-over time $\tau=L / U$ ). As explained in $\S 2$, the problem is divided into sub-problems in the time direction, each of size $2 \pi \times 2 \pi \times 4.2$, in order to fit the available computer memory. The coarsest level of the space-time grid is $m_{x} \times m_{y} \times m_{t}$, and is refined dyadically as needed such that at scale $j$ it is $m_{x} 2^{j} \times m_{y} 2^{j} \times m_{t} 2^{j}$. For all simulations $m_{x}=m_{y}=64$ and $m_{t}=2$, and we use three levels of refinement, so the finest uniform grid is $512 \times 512 \times 16$. The 


\begin{tabular}{lrcccc} 
Run & \multicolumn{1}{c}{ Re } & Resolution & $\Delta x$ & $\lambda$ & $R e_{\lambda}$ \\
I & 1260 & $192 \times 192$ & $3.3 \times 10^{-2}$ & $1.1 \times 10^{-1}$ & 138 \\
II & 2530 & $192 \times 192$ & $3.3 \times 10^{-2}$ & $8.3 \times 10^{-2}$ & 195 \\
III & 5050 & $192 \times 192$ & $3.3 \times 10^{-2}$ & $5.9 \times 10^{-2}$ & 275 \\
IV & 10100 & $256 \times 256$ & $2.5 \times 10^{-2}$ & $4.1 \times 10^{-2}$ & 389 \\
V & 20200 & $384 \times 384$ & $1.6 \times 10^{-2}$ & $2.9 \times 10^{-2}$ & 551 \\
VI & 40400 & $512 \times 512$ & $1.2 \times 10^{-2}$ & $2.0 \times 10^{-2}$ & 779
\end{tabular}

TABLE 1. Parameters for the reference pseudo-spectral DNS. The initial integral length scale $L \approx 1.09$ and the initial r.m.s. velocity is $U \approx 4.65$. $R e_{\lambda}$ is the Taylor Reynolds number, based on the initial Taylor scale $\lambda=\sqrt{E / E_{\omega}}$ (where $E$ is the kinetic energy and $E_{\omega}$ is the enstrophy.)

(a)

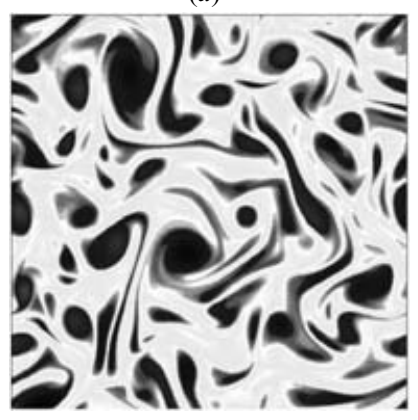

(b)

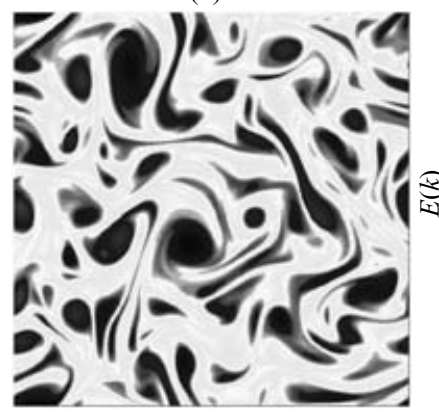

(c)

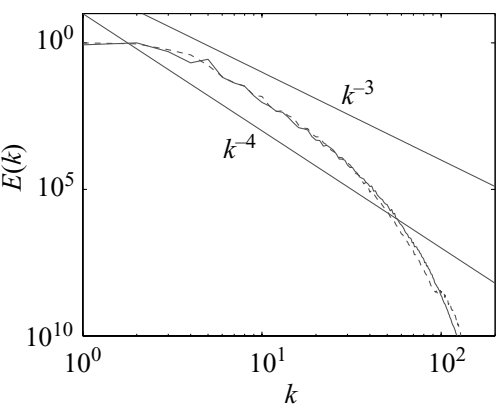

Figure 1. The vorticity field at $t=126$ eddy turn-over times and $R e=40400$. (a) Computed from an average 7895 spatial wavelet modes (total space-time modes in sub-domain $t=[123.8,128.0]$ divided by the number of time levels). (b) Computed from 263169 Fourier modes using the pseudo-spectral method. (c) Energy spectra: - - -, wavelet, - spectral.

grid sizes at the finest level are therefore $\Delta x \approx 1.2 \times 10^{-2}$ and $\Delta t \approx 2.6 \times 10^{-1}$. Note that the time scale of two-dimensional turbulence is believed to be the eddy turn-over time, independent of scale and Reynolds number (Davidson 2004). The simulations use a fixed error tolerance $\epsilon=10^{-4}$ (unless otherwise noted), which is small enough that we can consider the STAWCM to be a direct numerical simulation (DNS).

In order to verify the results of the STAWCM method, we calculated reference solutions at the same Reynolds numbers using a standard pseudo-spectral DNS (e.g. Vincent \& Meneguzzi 1991; Kevlahan \& Wadsley 2005). For a given Reynolds number, the minimum necessary resolution was determined by checking that the energy spectrum did not change significantly when the resolution was increased. As pointed out in the introduction, the Kolmogorov scale for two-dimensional turbulence is $O\left(R e^{-1 / 2}\right)$, which means the grid size $\Delta x \ll L R e^{-1 / 2}$ (where $L$ is the integral scale).

\section{Results}

We first check that the results of the STAWCM simulation agree with the reference pseudo-spectral simulation at $R e=40400$ (the highest Reynolds number considered). Figure $1(a, b)$ shows that the vorticity fields are very similar even though the STAWCM uses 33 times fewer modes spatially (on average) when $\epsilon=5 \times 10^{-4}$. Figure $1(c)$ shows that the energy spectra from the two simulations also agree. These 

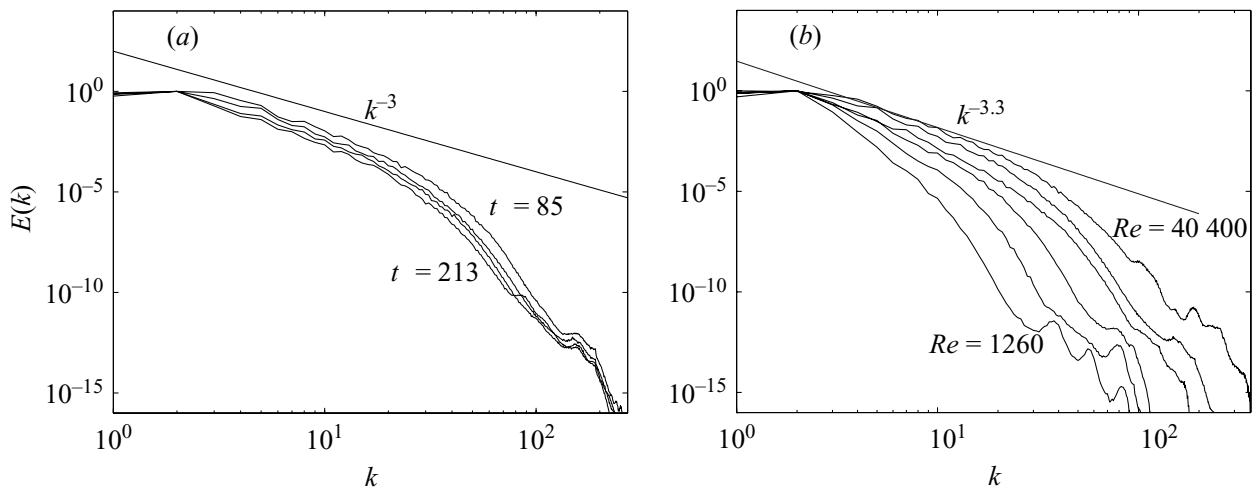

FigURE 2. Energy spectra. (a) $R e=20200$, at times $t=85,128,170,213$ compared with the initial spectrum $k^{-3}$. (b) $t=126$ for $R e=1260,2530,5050,10100,20200,40400$ compared with experimental spectrum $k^{-3.3}$.
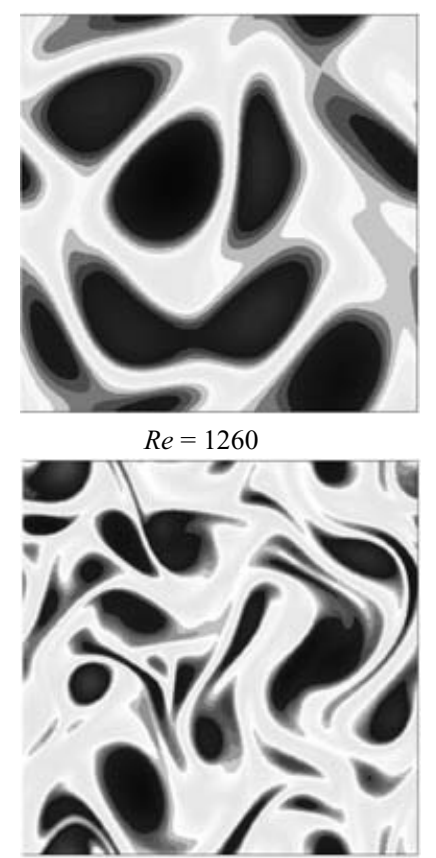

$\operatorname{Re}=10100$

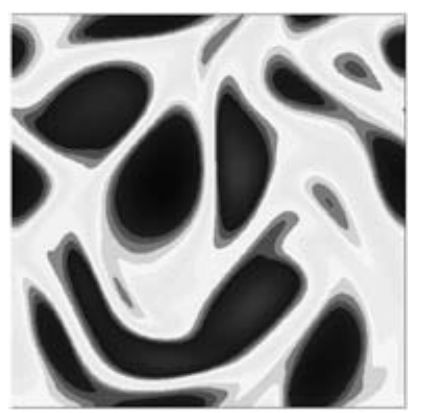

$\operatorname{Re}=2530$

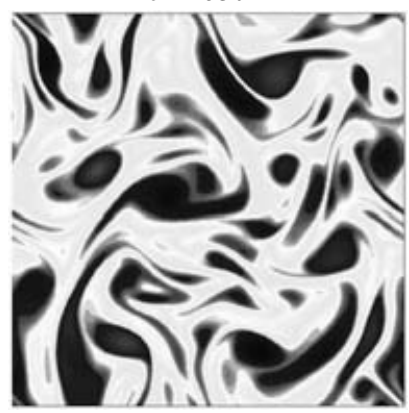

$R e=20200$

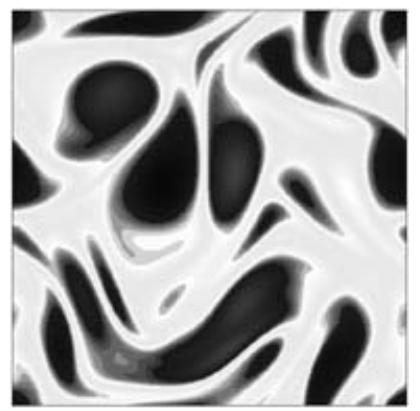

$\operatorname{Re}=5050$

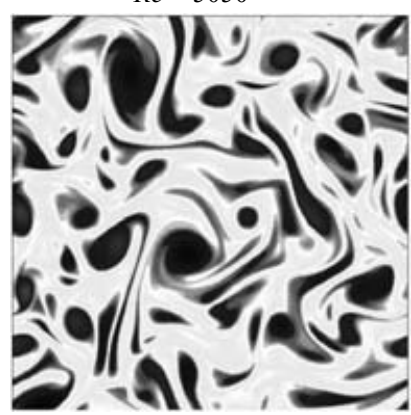

$R e=40400$

FIGURE 3. Vorticity field at $t=126$.

results confirm that the STAWCM is equivalent to standard pseudo-spectral DNS, but uses far fewer computational modes.

Figure $2(a)$ shows the time development of the energy spectrum at fixed Reynolds number $R e=20200$. Note that the spectrum does not change significantly at these intermediate times. Figure $2(b)$ shows how the energy spectrum at fixed time $t=126$ varies with Reynolds number. As expected, the power-law range increases and the slope decreases with Reynolds number. This plot also confirms that we have fully resolved all active length scales. Figure 3 shows how coherent vortices decrease in size, increase in number and are accompanied by intense filamentary structures as Reynolds 

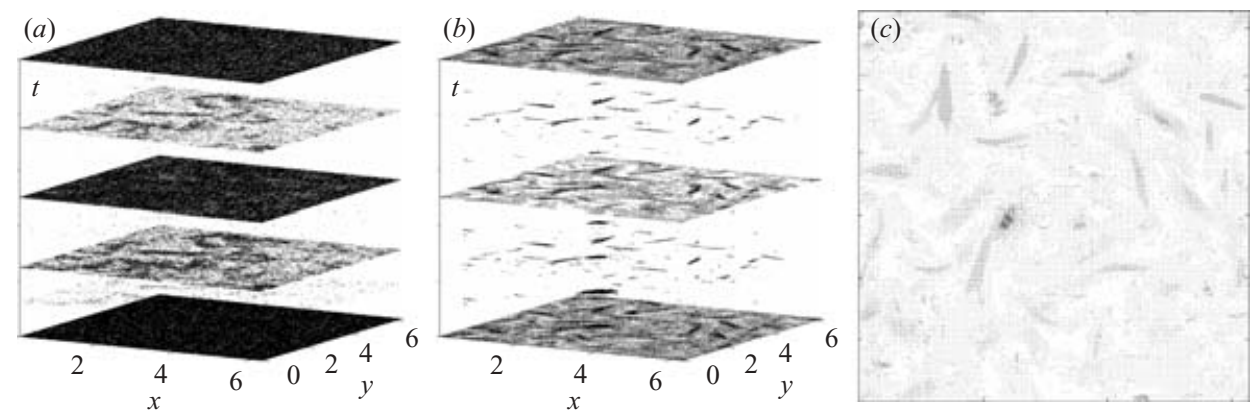

Figure 4. Adaptive wavelet grids at $R e=40400$ (half the time domain, i.e. 9 time levels, is shown). (a) Space-time grid: first time interval $t \in[0,2.1]$. (b) Space-time grid: final time interval $t \in[123.8,126.0]$. (c) Spatial grid only at $t=126.0$. Note the strong time intermittency of the solution: the smallest time step is strongly localized in space.
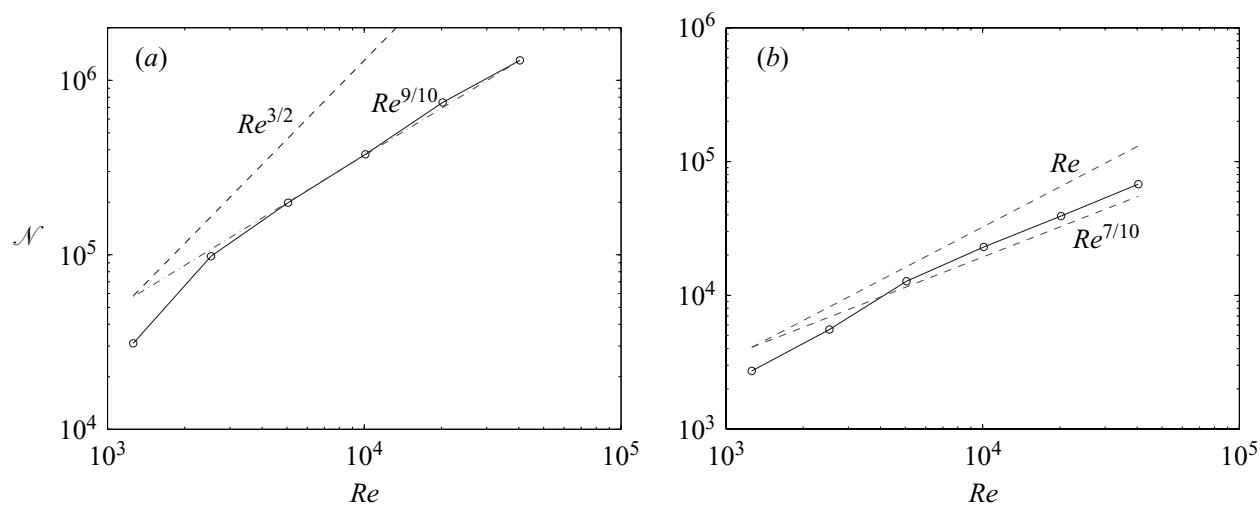

Figure 5. Degrees of freedom $\mathscr{N}$ as function of Reynolds number $R e$ compared with fitted slopes and the usual non-intermittent computational estimates. (a) Space-time. (b) Space only.

number increases. These changes are responsible for the increased intermittency of high-Reynolds-number turbulence. We will show that this intermittency significantly reduces the total number of degrees of freedom compared with the uniform-grid estimate $O\left(R e^{3 / 2}\right)$.

We now present the main results of this paper: on grid adaptation, wavelet compression and the number of space-time modes necessary to calculate decaying two-dimensional turbulence. We also estimate the scaling exponent $\alpha$ in equation (1.2).

Figure $4(a)$ shows the computational grid when the solution has converged (i.e. the residual error is less than $\epsilon$ ). Note that for this figure we use a slightly larger $\epsilon=5 \times 10^{-4}$. The results in figure 4 show the ability of the method to provide both an adaptive spatial grid and local time steps. It is clear from figure $4(a, b)$ that we do not actually use the smallest time step at every spatial location. The smallest time step is used only rarely, and only at those spatial locations where the solution has a strong gradient. Comparing figures $4(a)$ and $4(b)$ shows that the turbulence dynamics significantly increases intermittency compared to the random phase initial condition with a similar energy spectrum.

Figure $5(a)$ shows how the number of space-time degrees of freedom in a time interval of 21 eddy turn-over times $(107 \leqslant t \leqslant 128)$ scales as a function of Reynolds number. From this graph we estimate that $\alpha$ in equation (1.2) is about 0.9. This is 
much less than the usual estimate of 1.5 , which shows the importance of intermittency. We checked that the scaling exponent is roughly constant for times $21 \leqslant t \leqslant 128$. This suggests that the decaying turbulence results should be similar to results from stationary forced turbulence, at least for intermediate times. As noted earlier, and shown in figure 4, the major source of intermittency is temporal, rather than spatial.

It is important at this point to distinguish the minimum physical and computational time scales. Two- and three-dimensional turbulence are qualitatively different because the minimum time scale of two-dimensional turbulence is believed to be scale (and hence Reynolds number) independent, while the minimum time scale of threedimensional turbulence decreases like $R e^{-1 / 2}$ (Davidson 2004). However, the minimum computational time scale is inversely proportional to the minimum spatial scale, i.e. $\Delta t \sim 1 / l_{\min }$, which give the usual estimate for the number of space-time degrees of freedom: $O\left(R e^{3 / 2}\right)$ in two dimensions and $O\left(R e^{3}\right)$ in three dimensions.

Figure $5(b)$ shows the average number of spatial degrees of freedom for $40 \leqslant$ $t \leqslant 128$ calculated using a similar time marching AWCM (Kevlahan \& Vasilyev 2005). The scaling is $\alpha \approx 0.7$, compared with the non-intermittent estimate 1 . Thus, two-dimensional turbulence is in fact quite intermittent in space, as well as in time. This strong intermittency is somewhat surprising since two-dimensional turbulence is usually considered to be relatively homogeneous, and temporal intermittency has not been investigated.

The $\beta$-model described in the introduction implies that the spatial fractal dimension of decaying two-dimensional turbulence is $D_{F} \approx 1$.2. A simple extension of the $\beta$ model gives a temporal fractal dimension $D_{t} \approx 0.3$. Although these results depend on the assumption that the active fraction of the flow decreases like a power of the scale $l$, the fractal dimension does provide a qualitative measure of how intermittent the flow is. More sophisticated multi-fractal models have also been proposed.

\section{Conclusions}

We have used the space-time adaptive wavelet collocation method (Alam et al. 2006) to estimate the number of space-time computational modes $\mathscr{N}$ necessary to represent two-dimensional decaying turbulence as a function of Reynolds number. We find that $\mathscr{N} \sim R e^{0.9}$ for $1260 \leqslant R e \leqslant 40400$ over many eddy turn-over times, and that temporal intermittency is much stronger than spatial intermittency. In addition, we used a time marching AWCM (Kevlahan \& Vasilyev 2005) to estimate that the number of spatial computational modes scales like $R e^{0.7}$. This is the first time estimates of the number of degrees of freedom in turbulence have been obtained that fully include spatial and temporal intermittency. The $\beta$-model then implies that the spatial fractal dimension is $D_{F} \approx 1.2$, and the temporal fractal dimension is $D_{t} \approx 0.3$. These results suggest that the non-intermittent computational estimate of the number of space-time modes $\mathscr{N} \sim R e^{3 / 2}$ is not sharp for finite-precision adaptive numerical simulations. The relatively high compression confirms the importance of intermittency, and encourages the search for reduced mathematical models of twodimensional turbulence (e.g. in terms of coherent vortices).

We plan to extend the present results to three-dimensional turbulence, where nonintermittent estimates of the total number of computational space-time degrees of freedom vary between $O\left(R e^{3}\right)$ and $O\left(R e^{4}\right)$ (Yakhot \& Sreenivasan 2005).

N. K. was supported by NSERC, J. A. was supported by NSERC and SHARCNET and O.V. was partially supported by the NSF under grants No. EAR-0327269 and ACI-0242457. This support is gratefully acknowledged. This work was made possible 
by the facilities of the Shared Hierarchical Academic Research Computing Network (SHARCNET).

\section{REFERENCES}

Alam, J., Kevlahan, N. K.-R. \& Vasilyev, O. V. 2006 Simultaneous space-time adaptive wavelet solution of nonlinear partial differential equations. J. Comput. Phys. 214, 829-857.

Constantin, P. 1985 Attractors representing turbulent flows. Mem. Am. Math. Soc. 53 (314), 1-67.

Constantin, P., Foias, C. \& Temam, R. 1988 On the dimension of the attractors in two-dimensional turbulence. Physica D 30, 284-296.

Davidson, P. A. 2004 Turbulence: An Introduction for Scientists and Engineers. Oxford University Press.

Doering, C. R. \& Foias, C. 2002 Energy dissipation in body-forced turbulence. J. Fluid Mech. 467, 289-306.

Farge, M., Schneider, K. \& Kevlahan, N. K.-R. 1999 Non-Gaussianity and coherent vortex simulation for two-dimensional turbulence using an adaptive orthogonal wavelet basis. Phys. Fluids 11, 2187-2201.

FoIAS, C. \& PRODI, G. 1967 Sur le comportement global des solution non stationnaires des quations de Navier-Stokes en dimension deux. Rend. Sem. Math. Univ. Padova 39, 1-34.

Foias, C. \& Temam, R. 1984 Determination of the solutions of the Navier-Stokes equations by a set of nodal values. Math. Comput. 43, 117-133.

Frisch, U. 1995 Turbulence: The Legacy of A. N. Kolmogorov. Cambridge University Press.

Frisch, U., NelKin, M. \& Sulem, P.-L. 1978 A simple dynamical model of intermittent fully developed turbulence. J. Fluid Mech. 87, 719-736.

Friz, P. K. \& Robinson, J. C. 2001 Parametrising the attractor of the two-dimensional Navier-Stokes equations with a finite number of nodal values. Physica D 148, 201-220.

GALDI, G. P. 2006 Determining modes, nodes and volume elements for stationary solutions of the Navier-Stokes problem past a three-dimensional body. Arch. Rat. Mech. Anal. 180, 97-126.

Jones, D. A. \& Titi, E. S. 1993 Upper bounds on the number of determining modes, nodes and volume elements for the Navier-Stokes equations. Indiana Univ. Math. J. 42, 875-887.

Kevlahan, N. \& Vasilyev, O. 2005 An adaptive wavelet collocation method for fluid-structure interaction at high Reynolds numbers. SIAM J. Sci. Comput. 26, 1894-1915.

KeVlahan, N. K.-R. \& WadSley, J. 2005 Suppression of three-dimensional flow instabilities in tube bundles. J. Fluids Struc. 20, 611-620.

Martin, B. K., Wu, X. L., Goldburg, W. I. \& Rutgers, M. A. 1998 Spectra of decaying turbulence in a soap film. Phys. Rev. Lett. 80 (18), 3964-3967.

Paladin, G. \& Vulpiani, A. 1987 Degrees of freedom of turbulence. Phys. Rev. A 35, 1971-1973.

Tran, C. V., Shepherd, T. G. \& Cho, H. R. 2004 Extensivity of two-dimensional turbulence. Physica D 192, 187-195.

VASILYEV, O. V. 2003 Solving multi-dimensional evolution problems with localized structures using second generation wavelets. Intl J. Comput. Fluid Dyn. 17, 151-168.

Vasilyev, O. V. \& Kevlahan, N. K.-R. 2005 An adaptive multilevel wavelet collocation method for elliptic problems. J. Comput. Phys. 206, 412-431.

Vincent, A. \& MeneguzzI, M. 1991 The spatial structure and statistical properties of homogeneous turbulence. J. Fluid Mech. 225, 1-20.

Yakhot, V. \& SReEnivasan, K. R. 2005 Anomalous scaling of structure functions and dynamic constraints on turbulence simulations. J. Stat. Phys. 121, 823-841. 